\title{
Tratamiento multidisciplinar del traumatismo cervical con fractura de apófisis odontoides en militar, a propósito de un caso
}

\author{
Fuentes Estaban D. ${ }^{1}$, Navarro Suay R. ${ }^{2}$, Mestre Moreiro C. ${ }^{3}$, Fernández González J. ${ }^{4}$, \\ Villena Martín M. ${ }^{4}$
}

Sanid. mil. 2014; 70 (4): 274-278; ISSN: 1887-8571

\begin{abstract}
RESUMEN
La fractura de apófisis odontoides de la segunda vértebra cervical representa entre el 10 y el 15\% de todas las fracturas cervicales. En el medio militar, esta lesión puede ser originada por la práctica de deportes, actividades de especial riesgo como el paracaidismo y armas de fuego o artefacto explosivo. Un diagnóstico precoz y un adecuado tratamiento multidisciplinar pueden colaborar para el correcto manejo integral de una baja. Se ha realizado una revisión de la literatura en la base de datos del PUBMED, utilizado la palabra clave "fractura odontoides", "sedación", "Halo-Jacket".
\end{abstract}

PALABRAS CLAVE: fractura odontoides, militar, sedación.

Multidisciplinary treatment for cervical trauma with odontoid apophysis fracture in military member, a case report SUMMARY: odontoid fracture of the second cervical vertebra represents between 10 and $15 \%$ of all cervical fractures. In a military environment, this injury may be caused by the practice of sports, special risk such as skydiving and firearms or explosive device. Early diagnosis and appropriate multidisciplinary treatment can work for the proper management of a casualty. A literature review has been carried out in PUBMED database, using the keywords "fractura odontoides", "sedación", "Halo-Jacket".

KEYWORDS: odontoid fracture, military, Sedation.

\section{INTRODUCCIÓN}

Las fracturas de la columna cervical pueden provocar importantes secuelas neurológicas y originar la muerte del paciente. En concreto, las fracturas de la apófisis odontoides de la segunda vértebra cervical representan entre el 10\% y $15 \%$ de todas las fracturas de la columna cervical ${ }^{1,2}$. La sospecha diagnóstica precoz así como el manejo cuidadoso de la columna cervical durante todos los escalones sanitarios se consideran fundamentales para reducir la morbimortalidad de esta lesión ${ }^{3}$.

En países occidentales, la causa principal de esta lesión en la apófisis odontoides son los accidentes de tráfico, seguida de las caídas y de las los traumatismos deportivos. La flexión es el mecanismo de lesión más frecuentemente implicado. Según el trayecto, la fractura se clasifica en tres tipos. El tipo I afecta a la punta de la apófisis odontoides, es el más estable y no suele requerir tratamiento quirúrgico. La más frecuente es la tipo II, que atraviesan la base de la apófisis provocando la inestabilidad mayor. La tipo III, afectan al cuerpo del axis. Considerando el grado de estabilidad y el trayecto de la fractura, el tratamien-

${ }^{1}$ Cte. Médico. Escuela Militar de Sanidad / Hospital Central de la Defensa Gómez Ulla. Servicio de Medicina Intensiva.

${ }^{2}$ Cte Médico. Servicio de Anestesiología y Reanimación.

${ }^{3}$ Tcol. Médico. Servicio de Neurocirugía.

${ }^{4}$ Facultativo Especialista de Área. Servicio de Neurocirugía.

Hospital Central de la Defensa Gómez Ulla. Madrid. España.

Dirección para correspondencia: docalcala@hotmail.com

Recibido: 17 de diciembre de 2013

Aceptado: 26 de febrero de 2014 to puede ser conservador (inmovilizador Halo Jacket o collarín tipo Minerva) o quirúrgico ${ }^{1-3}$.

A continuación describimos el caso de un varón que durante un accidente de tráfico sufre un traumatismo cervical con fractura no desplazada de la apófisis odontoides de la segunda vértebra cervical. Después de descartar otras lesiones graves, se decide tratar la fractura de forma conservadora, colocando un dispositivo inmovilizador Halo Jacket con la intención de estabilizar la columna cervical hasta la consolidación final de la fractura.

\section{CASO CLÍNICO}

Antecedentes

Se presenta el caso de un militar de 27 años ( $76 \mathrm{Kg}, 175 \mathrm{~cm}$ ), con antecedente de anemia ferropénica de origen no filiado en tratamiento actual con hierro oral, que sufrió un accidente de tráfico cuando se dirigía hacia su lugar de trabajo. Se encontraba en el asiento del copiloto con el cinturón de seguridad abrochado cuando su vehículo colisionó lateralmente contra un camión.

\section{Manejo prehospitalario}

Una unidad de soporte vital avanzado prehospitalario extricó al paciente que se encontraba consciente y con dolor (EVA 8/10). Se le inmovilizó la región cervical mediante un collarín tipo Philadelphia, se le administró oxigenoterapia a $3 \mathrm{lpm}$, midazolam (1 mg i.v), fentanilo (50 microgramos i.v) y fluidoterapia 
de mantenimiento para realizar la evacuación hasta el Hospital Central de la Defensa Gómez Ulla por expreso deseo del herido.

\section{Primera valoración en el ámbito hospitalario.} Valoración secundaria. Primeros procedimientos

Tras el aviso recibido por parte del centro de coordinación prehospitalario, se preparó en el servicio de urgencias del hospital un equipo facultativo multidisciplinar (médico de familia, anestesiólogo, intensivista, cirujano general y radiólogo) apoyado por personal auxiliar. A su llegada, el paciente estaba consciente y orientado, refiriendo dolor (EVA 4/10) a nivel cervical, sin focalidad neurológica y con pupilas isocóricas reactivas. La auscultación pulmonar era normal con murmullo ventricular conservado, sin puntos dolorosos en parrilla costal ni esternal pero con dolor a nivel apófisis espinosas dorsales. El abdomen era blando, depresible, doloroso a exploración profunda a nivel de hipogastrio, con ruidos hidroaéreos conservados y sin signos de peritonismo. Movilizaba las cuatro extremidades. Presentaba dolor a palpación a nivel de rotula derecha acompañado de dolor a los movimientos activos con la flexoextensión sin presentar deformidad ni impotencia funcional.

Los resultados de la analítica de sangre estaban en rango de normalidad excepto el valor de CPK que ascendió a 1000 U/1. Los TAC craneal y torácico descartaron patología, sin embargo el TAC cervical mostró fractura incompleta de apófisis odontoides tipo III (Figura 1 y 2). Este hallazgo también apareció en la RM de columna cervical que descartó la presencia de signos de estenosis espinal o compromiso mieloradicular. El estudio radiológico se completó con la realización de una ecografía abdominal en la que no apareció líquido libre en pericardio, en peritoneo ni en otras estructuras abdominales.

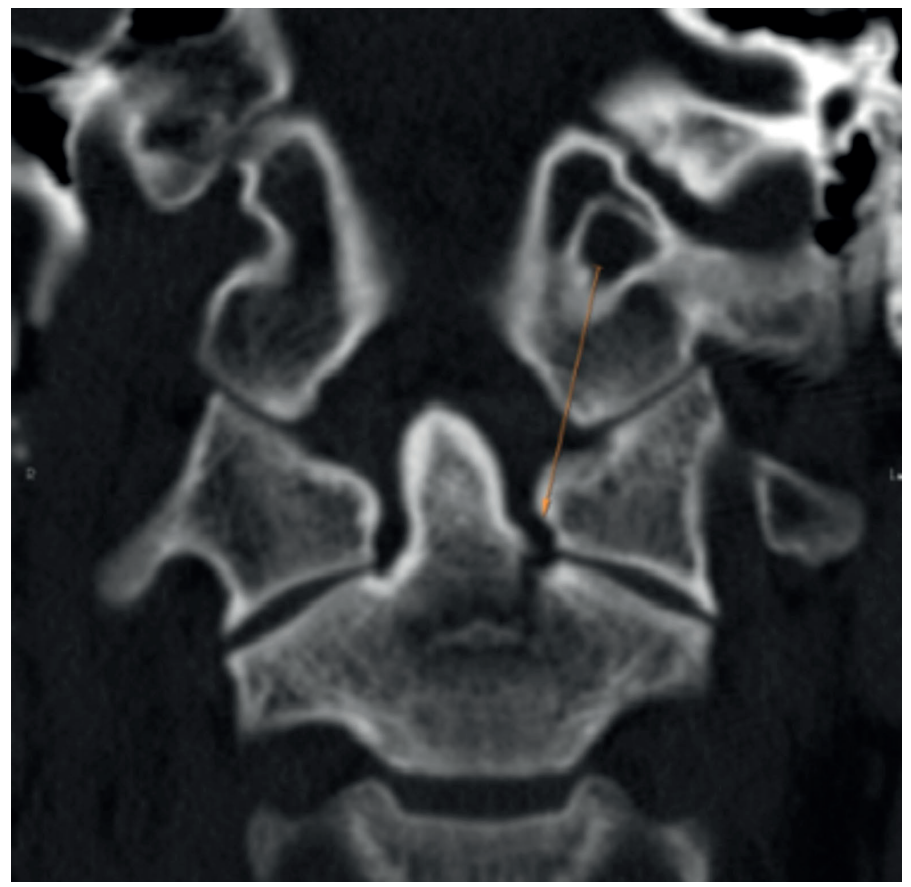

Figura 1. TAC cervical (vista coronal) en la que aparece la fractura de odontoides tipo III.
Tratamiento definitivo global: el manejo anestésico, neuroquirúrgico y psicosocial

Se consultó con el servicio de neurocirugía que decidió su ingreso en planta y mantenimiento del collarín tipo Philadelphia hasta la inmovilización definitiva mediante un dispositivo de fijación externa tipo Halo Jacket. A los 4 días del ingreso se procedió a la colocación de este fijador externo en quirófano. Con el objetivo de que el paciente pudiese colaborar en la colocación de la inmovilización y permitiese la realización de una radiografía transoral durante el procedimiento quirúrgico, se decidió sedoanalgesia empleando midazolam (dos bolos de $2 \mathrm{mg}$ iv), ketamina (dos bolos de $25 \mathrm{mg}$ iv) y anestesia local (lidocaína 2\%: $500 \mathrm{mg}$ ) en los puntos de anclaje de la fijación craneal. Para mantener una correcta posición en decúbito supino sobre la mesa de quirófano se colocó una tabla rígida desde la cabeza a los pies del paciente. La monitorización elegida fue grado I (frecuencia cardíaca, saturación de oxígeno, tensión arterial no invasiva y electrocardiograma), a la que se añadió capnografía (introduciendo en una coana un Abocath ${ }^{\circledR}$ número 14 parcialmente seccionado unido mediante el anclaje luer-lock a la línea del capnógrafo de la torre de anestesia) (Figura 3). Se fijó el Halo Jacket al cráneo mediante tornillos y se adaptó el chaleco del dispositivo al tórax del paciente gracias a unos anclajes laterales. El tiempo quirúrgico fue aproximadamente de 60 minutos, tras los cuales el paciente ingresó en la unidad de reanimación postquirúrgica en sedentación. Después de una hora fue dado de alta de esta unidad y pasó a la sala de ingreso de neurocirugía donde toleró la posición de decúbito supino, sedentación y progresivamente bipedestación. Durante la estancia hospitalaria se solicitaron interconsultas a anestesiología (que individualizó el tratamiento del dolor), psiquiatría (que le diagnosticó de "trastorno adaptativo tras accidente de tráfico" y recomendó sertralina, lexatín y zolpidem) y psicología (que en su entrevista le encontró con "ánimo subdepresivo, de componente ansioso, insomnio y presencia de pesadillas"). El paciente aceptó la colaboración del páter del hospital, se informó a la Dirección del centro, a sus

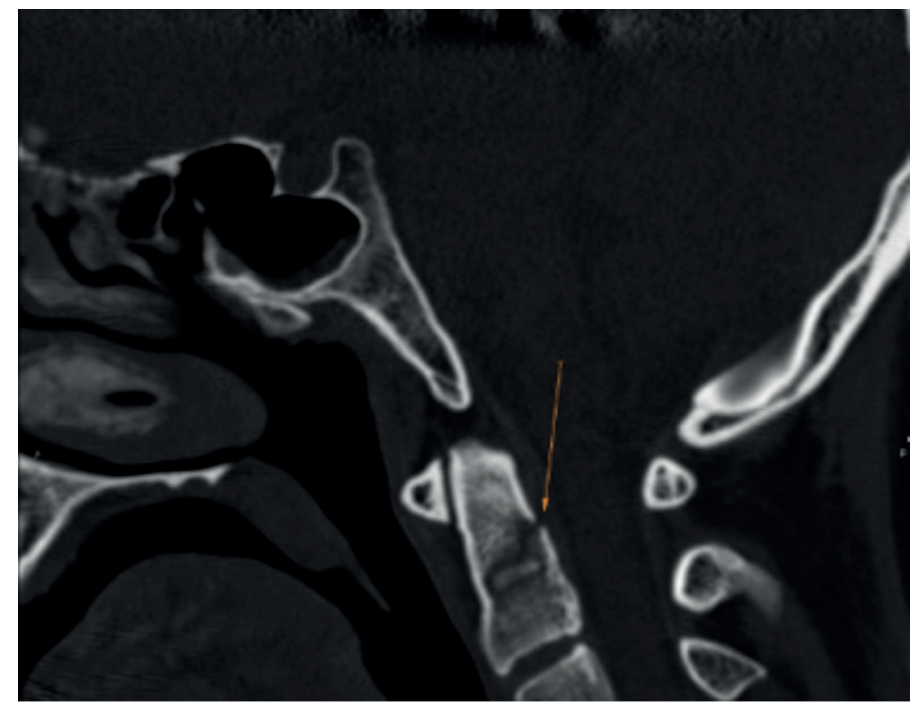

Figura 2. TAC cervical (vista sagital) en la que aparece la fractura de odontoides tipo III. 


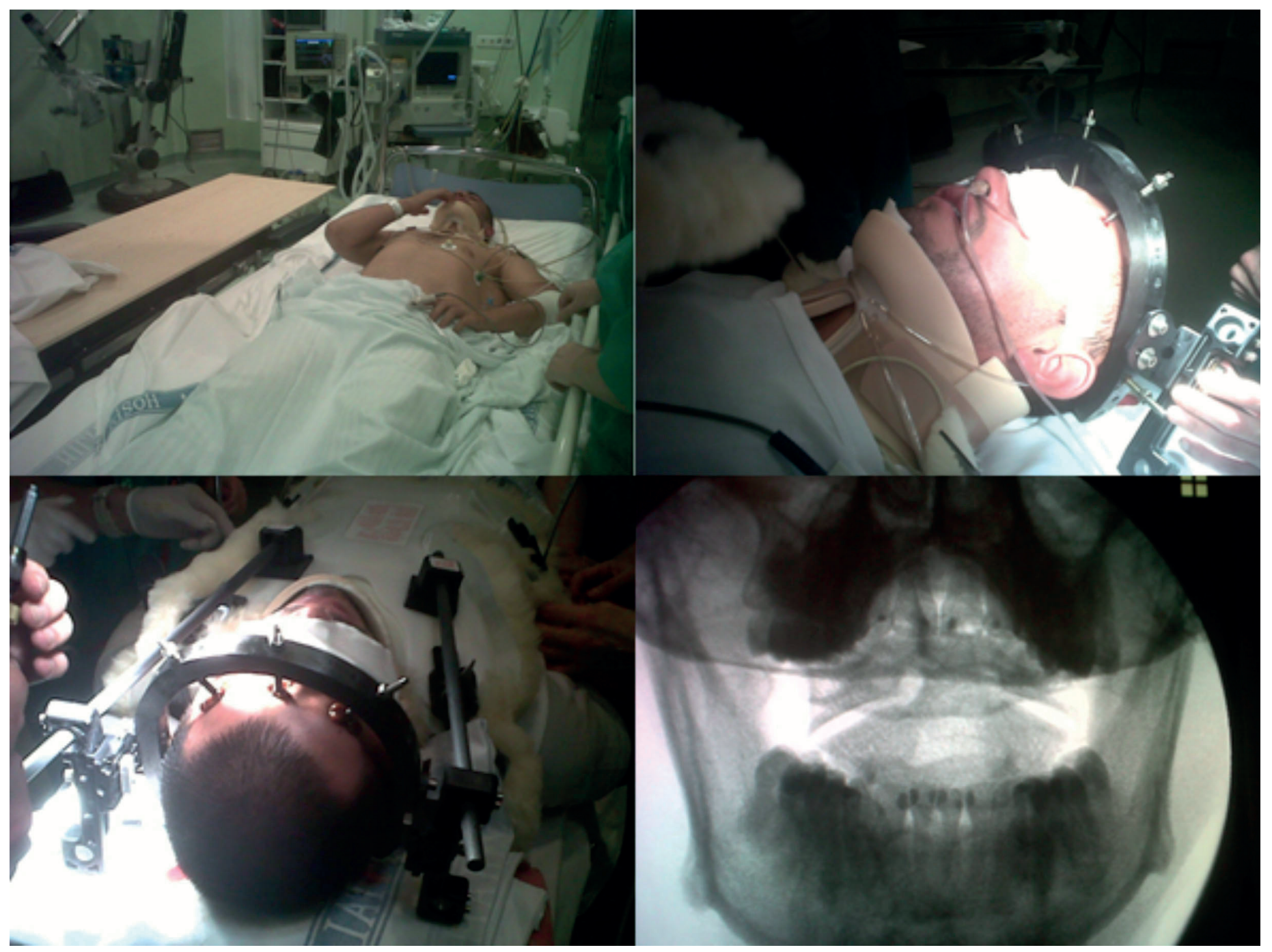

Figura 3. Distintos momentos del procedimiento quirúrgico. En la imagen de la esquina superior izquierda se observa el tablero espinal colocado sobre la mesa de quirófano. En la imagen de la esquina superior derecha aparece los tornillos de fijación y el detalle de la capnografía a nivel nasal. La imagen de la esquina inferior izquierda muestra la sujeción torácica. La imagen de la esquina inferior derecha es la radioscopia transoral para valorar la apófisis odontoides dentro del quirófano.

mandos directos así como a su unidad militar de procedencia (Brigada Paracaidista), se contactó con una residencia militar para que facilitase la estancia durante su convalecencia y por último se coordinó el apoyo familiar con la unidad de apoyo a heridos y familiares de fallecidos y heridos en acto de servicio de las Fuerzas Armadas.

\section{Manejo tras el alta hospitalaria}

Por mejoría clínica y tras corroborar mediante radiografías cervicales de control la buena evolución de la fractura de la apófisis odontoides, a los quince días el paciente fue dado de alta hospitalaria con revisión semanal por parte del servicio de neurocirugía durante 6 meses.

\section{DISCUSIÓN}

La fractura de apófisis odontoides: biomecánica, clasificación y manejo neuroquirúrgico

La articulación atloodontoidea es una diartrosis del genero trocoide, formada por dos partes: la parte anterior entre el arco anterior del atlas y la apófisis odontoides o articulación atloidoodontoidea propiamente dicha. La parte posterior entre la apófisis odontoides y el ligamento transverso o articulación sindesmoodontoidea. Una clasificación aceptada para las fracturas de la segunda vértebra cervical es la de Anderson y D'Alonzo que las divide en: fractura tipo I (avulsión del extremo del odontoide, sin consecuencia clínica), fractura tipo II (fractura de la base del odontoides; su tratamiento es orto- 
pédico, Minerva o Halo Jacket por 3 meses. Un 20\% termina en pseudoartrosis, que debe ser solucionada quirúrgicamente) y fractura tipo III (el trazo de fractura compromete el cuerpo del Axis. Consolida prácticamente siempre con tratamiento ortopédico). Es importante, para discernir entre el tratamiento ortopédico y quirúrgico, determinar la estabilidad de la fractura. Si la fractura es estable, el tratamiento de las lesiones es ortopédico. Sin embargo si es inestable, el tratamiento de elección es el quirúrgico ${ }^{1-3}$. A los 4 días del ingreso hospitalario se decidió colocar un Halo-Vest o Halo Jacket en quirófano. Este dispositivo es un collar sólido completo o no, anclado al cráneo con tornillos y sostenido por una abrazadera rígida que rodea los hombros, con conexiones ajustables que facilitan el control tridimensional de la relación hombros-cráneo ${ }^{3}$. Este inmovilizador puede ser utilizado aislado o en combinación con elementos de fijación. En nuestro caso se empleó un tablero espinal sobre la mesa del quirófano con el objetivo de alinear los ejes corporales involucrados en la colocación del Halo Jacket. En un principio se fijaron progresivamente los tornillos de anclaje del Halo en cráneo para a continuación colocar la pieza torácica y por último se anclaron los fijadores que unían ambas partes sin incidencias. Se empleó radioscopia de control durante este procedimiento para asegurar la correcta colocación del dispositivo.

\section{La importancia de esta lesión en el ambiente bélico}

La importancia de las lesiones cervicales en el personal militar es alta, debido a la gran actividad física que debe realizar, al medio donde puede desarrollar su actividad profesional, a las medidas de protección que se emplean y los a posibles ataques del enemigo con arma de fuego o artefactos explosivos. Hauret ${ }^{4}$ y Ressort ${ }^{5}$ describen que las lesiones deportivas que afectan a la región cervical oscilan entre 6-9\%. El militar pertenece a la Brigada Paracaidista y lleva realizados 27 saltos paracaidistas de tipo automático. Numerosos estudios del ejército estadounidense $^{6}$, británico ${ }^{7}$, francés ${ }^{8}$ y australiano ${ }^{9}$ muestran lesiones de distinta gravedad en el cuello de tropas paracaidistas. Sarabia ${ }^{10}$ analiza los traumatismos en 338 paracaidistas militares españoles, de ellos 5,9\% afectaron al cuello (2 graves y 18 leves). Aunque consideramos que es imposible averiguar si la experiencia del militar en saltos de tipo automático fue un factor de riesgo para la lesión cervical sufrida, lo más probable es que el paciente no pueda volver a realizar este tipo de actividad en un futuro. Los medios de protección también pueden influir en estas lesiones. Possley ${ }^{11}$ analiza las lesiones en columna vertebral en personal militar desplegado en Irak y Afganistán entre el 2001 y 2009, concluyendo que la región toracolumbar es la zona más afectada y destaca una menor incidencia de lesión en columna en las tropas que iban a bordo de vehículos blindados $(9 \%)$ respecto a los que van a pie (24\%). Por último los agentes lesionales (arma de fuego o artefacto explosivo) también pueden afectar a la región cervical. Un estudio estadounidense ${ }^{12}$ analiza 872 bajas en combate con lesiones en columna vertebral. E1 83\% fueron fracturas, siendo el explosivo el principal agente lesional en Afganistán mientras que Irak el agente más prevalente fue el arma de fuego.

\section{La importancia de la Imagen}

Múltiples han sido las pruebas radiológicas realizadas en este caso: TAC craneal, cervical, torácico y ecografía abdominal durante el ingreso, resonancia magnética preoperatoria, radioescopia intraoperatoria y finalmente radiografías cervicales de control postoperatorio. El TAC está considerado como método de elección para detectar lesiones en un paciente traumatizado estable con una estrategia donde se definen diferentes focos de interés: cerebro, columna, tejidos blandos del cuello, tórax/mediastino y abdomen ${ }^{13}$.

\section{El manejo anestesiológico}

Desde el punto de vista anestesiológico se debía mantener al paciente con el mejor control del dolor posible, consciente en quirófano para que pudiera colaborar durante el procedimiento quirúrgico, con un complicado manejo de la vía aérea en caso de depresión respiratoria intraoperatoria por la presencia de los fijadores externos cervicales, una dificultad para la monitorización del grado de hipnosis y en caso de aparecer parada cardiorespiratoria una compleja realización de las maniobra de reanimación cardiopulmonar por la presencia del dispositivo de fijación a nivel torácica (Figura 4). Para la sedoanalgesia consciente se empleó midazolam y ketamina en bolo intravenoso como coadyuvantes a la anestesia local con lidocaína en los puntos de anclaje del dispositivo. La utilización de estos fármacos en ambiente militar han sido ampliamente descritos por la ventaja de conseguir ansiolisis y amnesia anterógrada (midazolam) con un grado de hipnosis y analgesia adecuado optimizando las condiciones hemodinámicas del paciente (ketamina $)^{14,15}$. Para el control de la vía aérea se prepararon como métodos de rescate una mascarilla laríngea Ambú Aura $\mathrm{I}^{\circledR}$ (Ambú, Madrid) número 4 y mascarilla LMA Fastrach ${ }^{\circledR}$ (LMA C Trach ${ }^{\mathrm{TM}}$, San Diego,CA) número 4. La presencia de tornillos fijados en la frente impedía el empleo de monitorización de hipnosis mediante índice biespectral $\mathrm{BIS}^{\circledR}$ disponible en el hospital. Como alternativa se optó por capnografía (sin ventilación mecánica) asumiendo que esta monitorización analiza la relación ventilación/perfusión, y que aunque útil, tan solo puede inferir el grado de hipnosis. Por último, en caso de parada cardiorespiratoria el dispositivo Halo Jacket cuenta la posibilidad de permitir el masaje cardiopulmonar gracias a una apertura centrotorácica en el citado inmovilizador.

\section{El manejo de las secuelas. Tratamiento psiquiátrico y rehabilitador}

El paciente fue diagnosticado de trastorno adaptativo tras sufrir el accidente de tráfico y precisó tratamiento psiquiátrico con sertralina, lexatin y zolpidem por vía oral. Esta intervención fue apoyada por el servicio de psicología que mediante entrevista psicológica encontró al paciente con ánimo subdepresivo, componente ansioso, insomnio y presencia de pesadillas. El apoyo prestado por el páter del hospital fue muy reconfortante para el enfermo posiblemente a consecuencia de sus fuertes conviccio- 
nes religiosas. A lo largo de la convalecencia estos signos clínicos mejoraron notablemente y no requirieron de más intervenciones en la esfera psiquiátrica, psicológica y emocional posiblemente pudiendo influir el inicio precoz de este tratamiento ${ }^{16,17}$.

Desde el comienzo de la atención hospitalaria y preservando el derecho de privacidad del paciente se fue informando a la dirección del hospital y a sus mandos militares directos. La intención fue facilitar la tramitación administrativa por parte de la primera sección de personal de su destino. Así mismo el servicio de sanidad de su unidad visitó periódicamente al enfermo en el hospital. Especial mención merece la colaboración recibida por parte de la Unidad de Apoyo a heridos y familiares de fallecidos y heridos en acto de servicio de las Fuerzas Armadas. Esta unidad se creó en 2010 para proporcionar un apoyo continuado a bajas y familiares ${ }^{18}$. En nuestro caso facilitó una ayuda económica para el entorno familiar del paciente y la coordinación con una residencia militar para que el enfermo pudiera alojarse hasta la resolución del cuadro.

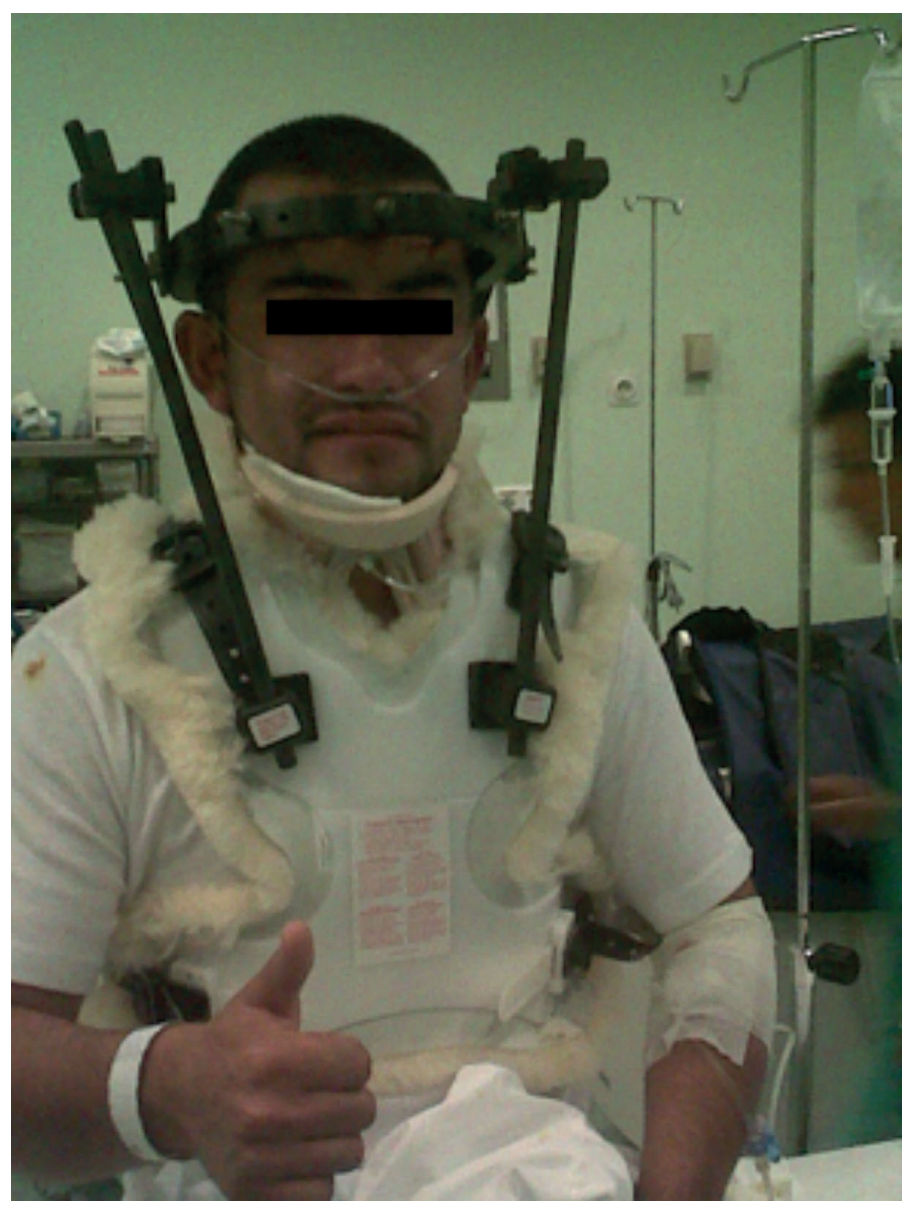

Figura 4. Inmovilización completa del Halo-Jacket con buena tolerancia por parte del paciente. En el centro del dispositivo aparecen las instrucciones a realizar en caso de necesitar la realización de maniobras de reanimación cardiopulmonar.

\section{CONCLUSIÓN}

Tras la revisión bibliográfica de las lesiones con fractura de apófisis odontoides se concluye que es una lesión muy poco frecuente en nuestro medio y representa entre el 10 y el 15\% de todas las fracturas cervicales. Está muy relacionada con la práctica de deportes, actividades de especial riesgo como el paracaidismo y armas de fuego o artefacto explosivo. Por ello un diagnóstico precoz y un adecuado tratamiento multidisciplinar como el realizado en este caso, pueden colaborar en la realización de un correcto manejo integral a una baja, fin último de la sanidad militar.

\section{BIBLIOGRAFÍA}

1. García MC. Aparatos de estabilización y de reemplazo de uso corriente en artrodesis y artroplastias de la columna cervical. Rev. Chilena de radiología 2008; 14 (4): 181-99.

2. Steltzlen C, Lazennec JY, Catonné Y, Rousseau MA. Unstable odontoid fracture: surgical strategy in a 22 case series, a literatura review. Orthop Traumatol Surg Res 2013; 99(5): 615-23

3. France JC, Powel EN, Emery SE, Jones DL. Early morbidity and mortality associated with elderly odontoid fractures. Orthopedics 2012; 35(6):889-94

4. Hauret KG, Jones BH, Bullock SH, Canham-Chervak M, Canada S. Musculoskeletal injuries description of an under-recognized injury problem among military personnel. Am J Prev 2010; 38(1): 61-70.

5. Ressort T, Desieux G, Marsan P, Thevenin-Garrot V. Sport related injuries and conditions. Sante Publique 2013; 25(3):263-70.

6. Craig SC. Parachuting injury surveillance, Fort Bragg, North Carolina, May 1993 to December 1994. Milit Med 1996; 162: 162-4.

7. Bricknell M, Craig S. Military parachuting injuries: a literature review. Occup. Med 1999;49:17-26.

8. Mayet A, Bay C, Salivas A, Verret C. Accident de parachutisme dans les unites aéroportées de la Région Terre Sud-ouest 2004-2005. Médecine et Armées 2009; 37:3-9.

9. Hughes $\mathrm{C}$, Weinrauch P. Military static line parachute injuries in australian comando battlalion. ANZ J Surg 2008;78:848-52.

10. Sarabia JM, Fernández J, Carreño L, Clavel M, Canteras M. Lesiones traumáticas paracaidistas. Revista de Medicina Militar 1993; 49(1): 57-60.

11. Possley DR, Blair JA, Freedman BA, Schoenfeld AJ, Lehman RA, Hsu JR. The effect of vehicle protection on spine injuries in military conflict. Spine $\mathrm{J}$ 2012;12(9):843-8.

12. Schoenfeld AJ, Laughlin MD, MCCriskin BJ, Bader JO, Waterman BR, Belmont PJ. Spinal injuries in United States military personnel deployed to Iraq and Afghanistan: an epidemiological investigation involving 7877 combat casualties from 2005 to 2009. Spine 2013;38(20):1770-8.

13. Martí M, Artigas JM, Vicente A, Carreras M. Manejo radiológico del paciente politraumatizado, evolución histórica y situación actual. Radiología 2010;52(2):105-14.

14. Dawes RJ, Mellor A. Pre-hospital anaesthesia. J R Army Med Corps 2011;156 (4): 289-94.

15. Mercer SJ. The drug of war, a historical review of the use of ketamine in military conflicts. J Royal Naval Medical Service 2009; 95(3): 145-50

16. Gould, M., Greenberg, N. and Hetherton, J. Stigma and the military: evaluation of a PTSD psychoeducational program. Journal of Traumatic Stress 2007; 20: 505-5

17. Iversen, A., Dyson, C., Smith, N., Greenberg, N.,Walwyn, R., Unwin, C., etal. 'Goodbye and good luck': the mental health needs and treatment experiences of British ex-service personnel. British Journal of Psychiatry 2005; 186: 480-6.

18. http://www.defensa.gob.es/info/servicios/unidaddeapoyo/ [Disponible 6 diciembre 2013]. 